\title{
Comparison of the therapeutic effects of two recombinant erythropoietin beta, biosimilar and reference formulations in patients with chronic kidney disease under hemodialysis
}

\author{
Maria del Carmen Popoca-Martínez ${ }^{1}$, Julio Flores-Garnica ${ }^{1}$, Odette Díaz-Avendaño ${ }^{1}$, \\ Emmanuel Canales-Vázquez ${ }^{2}$, Raúl Meixueiro-Montes De Oca \\ ${ }^{1}$ Nephrology Department, General Hospital “Dr. Darío Fernández Fierro” ISSSTE, Av. Revolución 1182, Col. San José Insurgentes, \\ México City 03900, Mexico \\ ${ }^{2}$ Clinical Research Department, Laboratorios PISA SA de CV. Miguel Ángel de Quevedo 555, Colonia Romero De Terreros, México City \\ 04310 Mexico
}

\section{Email address:}

jmeixueiro@pisa.com.mx (Meixueiro-Montes D. O. R.)

\section{To cite this article:}

Maria del Carmen Popoca-Martínez, Julio Flores-Garnica, Odette Díaz-Avendaño, Emmanuel Canales-Vázquez, Raúl Meixueiro-Montes De Oca. Comparison of the Therapeutic Effects of Two Recombinant Erythropoietin Beta, Biosimilar and Reference Formulations in Patients with Chronic Kidney Disease under Hemodialysis. Clinical Medicine Research. Vol. 3, No. 5, 2014, pp. 136-141.

doi: 10.11648/j.cmr.20140305.15

\begin{abstract}
Anemia is common in patients with chronic kidney diseases under hemodialysis and it's managed with recombinant erythropoietin formulation. The objective of the study is to compare the safety and efficacy of two recombinant erythropoietin formulations in patients undergoing hemodialysis with chronic kidney diseases. In this randomized, controlled, prospective, parallel open study 70 patients were treated for 24 weeks with either reference $\beta$ recombinant erythropoietin $(100 \mathrm{IU} / \mathrm{kg})$ or biosimilar $\beta$ recombinant erythropoietin $(100 \mathrm{IU} / \mathrm{kg})$. The primary efficacy endpoint was the hemoglobin and hematocrit levels from baseline to 24 week of treatment. The secondary endpoints were safety, weekly doses of both erythropoietins required to maintain hemoglobin levels and immunogenicity. There was no significant difference between the two preparations in terms of hemoglobin and hematocrit levels achieved. The weekly doses of both erythropoietins required to maintain hemoglobin levels were the same in both groups. The frequency of adverse events was similar in the two groups of treatment. Two patients of the reference erythropoietin group developed anti-erythropoietin antibodies. The biosimilar erythropoietin was comparable since the safety and efficacy point of view with the innovator erythropoietin in hemodialysis patients based on hemoglobin changes. The biosimilar erythropoietin when administered subcutaneously will be equally efficacious and may be interchangeable as therapy.
\end{abstract}

Keywords: Biosimilar rHuEPO, Safety, Efficacy, Renal Anemia, Hemodialysis

\section{Introduction}

Erythropoietin is a protein of 165 amino acids four of which are glycosylated [1] and actually is available in a recombinant form. In adult's humans, over $90 \%$ of erythropoietin is synthesized by kidneys cells, where its secretion is stimulated by oxygen deficiency. Erythropoietin exerts its effects by binding irreversibly to erythrocyte progenitor cells in the red bone marrow and the spleen. This results in increase of blood hemoglobin and consequently an increased oxygen supply to the kidneys.
Chronic kidney disease (CKD) is a prevalent and increasing health concern worldwide, and anemia is a frequent complication of CKD [2, 3]. The main cause of anemia in patients with CKD is insufficient synthesis of erythropoietin by the damaged kidney. Since the 1980s, erythropoiesis-stimulant agents have been used to treat CKD-associated anemia. Anemia management with erythropoiesis stimulants is a successful and well-tolerated therapy that has been demonstrated to improve quality of life a positively influence outcomes $[4,5]$. More than $90 \%$ of the end-stage renal disease patients require exogenous 
erythropoietin or transfusion to achieve and maintain target hemoglobin values [6] because of decreased endogenous erythropoietin production.

The treatment of the anemia of chronic renal failure with exogenous recombinant human erythropoietin (rHuEPO) is a well-established practice that has led to improvements in a variety of clinical parameters, including exercise tolerances $[7,8]$, quality of life and decreased mortality $[9,10]$.

In other hand, the ability to achieve and maintain target hemoglobin levels is complicated by a variety of mediating factors that impact responsiveness to erythropoietin, including comorbidities, inflammation, malnutrition and the anti-erythropoietin antibodies develop $[11,12]$.

Reports in hemodialysis patients indicate an effective erythropoiesis increment, ceasing or decrease of transfusion frequency [2, 3, 8-10]. However, this treatment is still expensive, not affordable for the National Health Systems in developing countries if an extensive use, for all patients who need it. The biosimilar could be similar from physicochemical properties but it is possible found differences in pharmacodynamics or pharmacokinetic properties that could have clinical repercussions. For this reason in necessary a clinical evaluation of the biosimilar against the reference drug.

Whether multiorigin recombinant proteins can be clinically interchangeable despite coming from different strains and manufacturing processes is a controversial matter nowadays $[13,14]$. If this principle is accepted, then a study that shows pharmacological similarity between two drugs would be enough to indicate its therapeutic interchangeability, given that are biological and pharmaceutically equivalent as well. The aim of this study was to assess the efficacy and safety of biosimilar $\beta$ rHuEPO versus innovator $\beta$ rHuEPO, administered subcutaneously three times a week for the treatment of anemia in hemodialysis patients with CKD.

\section{Methods}

This open randomized, parallel, prospective, comparative study enrolled 70 patients with CKD. Were eligible patients with 18-70 years, weighing 50-90 $\mathrm{Kg}$ both sexes, with anemia, hemoglobin $\leq 10 \mathrm{~g} / \mathrm{dL}$ and hematocrit $\leq 30 \%$ due to end-stage renal disease on hemodialysis ( 3 sessions per week), creatinin clearance rates $\leq 15-30 \mathrm{~mL} / \mathrm{min}$ in $24 \mathrm{hrs}$ urine collection who gave their written consent to participate. Other inclusion criteria were body mass index of 19-28 $\mathrm{kg} / \mathrm{m}^{2}$, reticulocytes $\leq 3 \%$ at screening, normal or only minor deviation in iron parameters (iron deficiency was defined as ferritin $<10 \mathrm{ng} / \mathrm{mL}$ ). The exclusion criteria were previous rHuEPO treatment, pregnancy, sepsis or active infection, cancer, hormonal treatment (except thyroid hormone, contraceptive or insulin), liver disease, uncontrolled hypertension, thrombocytopenia (platelet count $<75,000 / \mathrm{mm}^{3}$ ), severe psychiatric dysfunction, signs of active bleeding. They were withdrawn from trial if they abandoned voluntary, had severe adverse reactions, were subjected to renal transplant, used not allowed medication or other rHuEPO formulations.

The study was conducted in accordance with the Declaration of Helsinki, Good Clinical Practice and the study was approved by the ethics committee of the institution participant and all the patients gave their written informed consent.

Eligible subjects were randomized and allocated in two groups of treatment to receive one of the two different treatments three times a week for 24 weeks by intravenous injection. The treatment with biosimilar $\beta \mathrm{rHuEPO}$ consisted of injections of $100 \mathrm{IU} / \mathrm{kg}$ body weight (Exetin, Laboratorios PiSA, Guadalajara, México). The reference $\beta$ $\mathrm{rHuEPO}$ treatment consisted of injections of $100 \mathrm{IU} / \mathrm{kg}$ body weight (Recormon, Roche, Switzerland). This dose was maintained for 2 weeks with subsequent titration to maintain hemoglobin at $10-12 \mathrm{~g} / \mathrm{dL}$. Titration was as follows: dose was increased by $50 \%$ if hemoglobin dropped below $10 \mathrm{~g} / \mathrm{dl}$ or decreased by $25 \%$ if hemoglobin was above $12 \mathrm{~g} / \mathrm{dL}$. Dose was also altered if a patient's dry weight changed by more than $5 \mathrm{~kg}$. Dose adjustment based on hemoglobin level was only performed once in ever four week period. Both $\beta$ $\mathrm{rHuEPO}$ were supplied as $10,000 \mathrm{IU} / \mathrm{mL}$ formulations. Subjects fasted for at least $8 \mathrm{~h}$ prior to and $2 \mathrm{~h}$ after administration.

Blood samples for hemoglobin and hematocrit concentration, were drawn on day 1 st and 2, 4, 6, 8, 10, 12, $14,16,18,20,22$, and 24 weeks, $10 \mathrm{~min}$ pre-dose. For determinations of transferring, ferritin, serum iron concentration, electrolytes ( $\mathrm{Na}, \mathrm{Cl}, \mathrm{P}, \mathrm{K}, \mathrm{Ca}, \mathrm{Mg}$ ), venous blood samples of $12 \mathrm{ml}$ were drawn on the first day and the 4 , $8,12,16,20$ and 24 weeks at the time dosing. For anti-erythropoietin antibodies assays in serum, a blood sample of $3 \mathrm{ml}$ was drawn on the $1^{\text {st }}$ day and the week 24 . All samples were analyses and performed in the same laboratory. The anti-erythropoietin antibodies assays were performed in Nanopharmacia Diagnostica Laboratory (Mexico City, Mexico).

The adverse events were obtained from pre and post-hemodialysis and spontaneous reporting by the subjects or from responses to non-leading questions from the clinical staff.

The primary efficacy endpoint was average hemoglobin and hematocrit levels over 4, 8, 12, 16, 20 and 24 weeks. Secondary analysis included: rHuEPO beta dose levels, the proportion of patient's hemoglobin values over $10 \mathrm{gr} / \mathrm{dl}$ and hematocrit values over 30\% during week 24 .

To test the homogeneity between the treatment groups, the Mann-Whitney's test was applied for quantitative control variables and the Fisher's exact test for the qualitative ones. The hematological variables were assessed every 4 weeks. To compare formulations the analysis of variance (ANOVA) and the estimation of confidence intervals $(90 \%)$ of the ratio were used. Laboratory variables were treated using paired analysis (Wilcoxon's test). Adverse reactions frequencies were compared between formulations using the Fisher's exact test. Anti-erythropoietin antibodies were screened for by validated ELISA on samples collected at baseline and the 
end of the 24 weeks of study.

\section{Results}

Seventy patients from General Hospital "Darío Fernández", ISSSTE, were recruited where they regularly received hemodialysis treatment. The demographic and baseline characteristics of the patients are shown in the Table 1. Age $(53.9 \pm 14.8$ vs $55.8 \pm 13.25$ years old, innovator and generic, respectively) and weight $(64.01 \pm 12.19$ vs $63.48 \pm$ $12.4 \mathrm{~kg}$, reference and biosimilar, respectively) were highly variable within each group. Overall ages ranged from 18 to 80 years and weights from 42 to $96 \mathrm{~kg}$. The groups were balanced regarding sex ( 21 female and 14 male vs 19 female and 16 male, innovator and generic, respectively). All the patients had specified the cause of renal failure, prevailing diabetes mellitus and hypertension. The hypothesis of homogeneity between the groups was accepted.

Table 1. Demographic data of both groups of patients (biosimilar and reference). No significant differences were found between both groups. SP= sistolic pressure; $D P=$ diastolic pressure; $H R=$ heart rate; $R F=$ respiratory frequency.

\begin{tabular}{llll}
\hline Variables & rHuEPO biosimilar & rHuEPO reference & Significance \\
\hline Age & $55.8 \pm 13.25$ years & $53.94 \pm 14.8$ years & \\
Sex & & & \\
Female & 19 & 14 & \\
Male & 16 & $64.1 \pm 12.19 \mathrm{~kg}$ & $p=0.15$ \\
Weight & $63.48 \pm 12.4 \mathrm{~kg}$ & $1.63 \pm 0.81 \mathrm{mts}$ & $p=0.85$ \\
Height & $1.65 \pm 0.53 \mathrm{mts}$ & $127.6 \pm 12.1 \mathrm{mmHg}$ & $p=0.55$ \\
SP & $129.77 \pm 12.7 \mathrm{mmHg}$ & $73.71 \pm 5.0 \mathrm{mmHg}$ & $p=0.69$ \\
DP & $70.14 \pm 6.9 \mathrm{mmHg}$ & $76.17 \pm 6.2 \times \mathrm{min}$ & $p=0.52$ \\
HR & $75.94 \pm 7.1 \times \mathrm{min}$ & $19.08 \pm 3.2 \times \mathrm{min}$ \\
RF & $19.94 \pm 2.1 \times \mathrm{min}$ & $P=0.44$ \\
\hline
\end{tabular}

The average doses at baseline was 4,000 IU for both groups and the average during the 24 weeks of treatment was 5,914 IU and 5,742 IU for reference and biosimilar respectively. No significant differences were found between both groups $(\mathrm{p}=0.85)$.

Overall mean \pm SD hemoglobin at baseline were $8.97 \pm$ $1.08 \mathrm{~g} / \mathrm{dL}$ vs $9.02 \pm 1.54 \mathrm{~g} / \mathrm{dL}$ and mean hematocrit $26.77 \pm$ $3.0 \%$ vs $27.19 \pm 4.52 \%$, for reference and biosimilar, respectively. There were no significant differences in both groups at baseline since the hemoglobin or hematocrit point of view ( $\mathrm{p}=0.43$ ) (Figure 1 and 2).

At the week 24 mean \pm SD hemoglobin and hematocrit were $12.24 \pm 0.99 \mathrm{~g} / \mathrm{dL}$ and $35.66 \pm 2.48 \%$ for reference $\mathrm{rHuEPO}$ and $12.57 \pm 1.28 \mathrm{~g} / \mathrm{dL}$ and $35.26 \pm 2.31 \%$ for biosimilar rHuEPO. The average levels of hemoglobin and hematocrit at weeks $4,8,12,16,20$ and 24 are shown in Figure 1 and 2 . There were no marked differences between groups $(p=0.34$ and $p=0.16$, for hemoglobin and hematocrit, respectively).

Over week 24, 94.28\% and $91.42 \%$ were over the predefined lower limits of $10 \mathrm{~g} / \mathrm{dL}$ for hemoglobin levels for reference and biosimilar group, respectively (Figure 3).

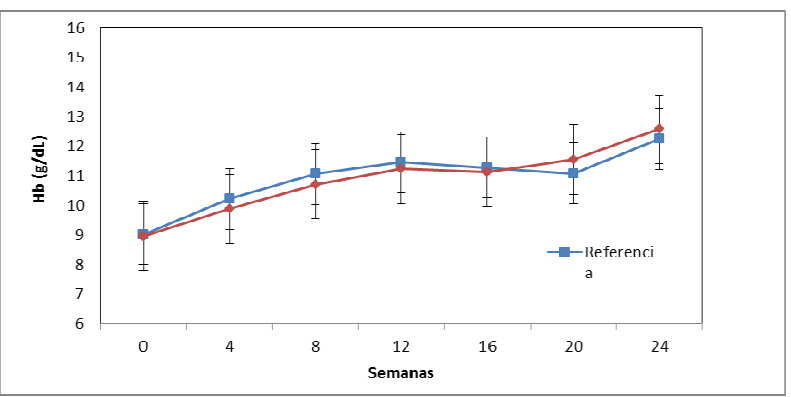

Figure 1. The primary efficacy endpoint (hemoglobin) during 24 weeks of treatment. No significant differences were found between both groups $(p=0.34)$.

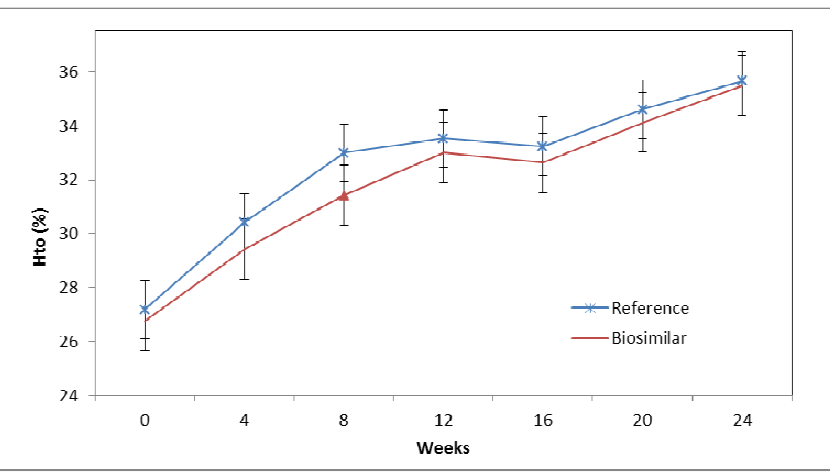

Figure 2. Levels of hematocrite during the 24 weeks of treatment. There were not significant differences between $\mathrm{rHuEPO}$ reference and biosimilar $(p=0.16)$.

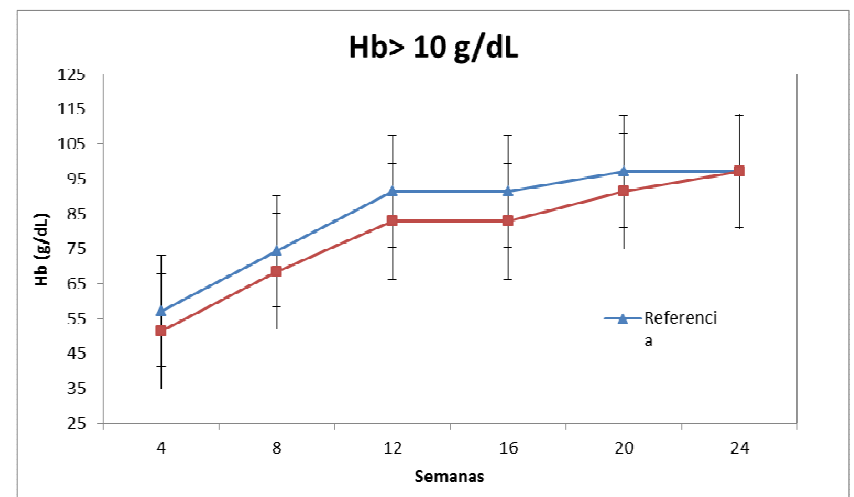

Figure 3. Percentage of patients of both groups that reach $\mathrm{Hb}>10 \mathrm{~g} / \mathrm{dL}$ at the end of the study.

With reference to $\mathrm{Na}, \mathrm{Cl}, \mathrm{K}, \mathrm{P}, \mathrm{Ca}$ and $\mathrm{Mg}$ electrolytes were not found significant differences between the groups during the 24 weeks of treatment.

After both treatments the transferrin concentration showed only a minor increase compared to baseline at weeks 16 and 24 , from $2.36 \pm 0.35$ to $2.43 \pm 0.28$ and $2.42 \pm 0.31$, 
respectively after $\mathrm{rHuEPO}$ reference group and from $2.41 \pm$ 0.26 to $2.42 \pm 0.38$ and $2.44 \pm 0.19$, respectively after rHuEPO biosimilar group. No changes were observed in ferritin and serum iron concentrations.

Mean changes in laboratory analysis, vital signs ad body weight from baseline to endpoint were minimal and there was no tendency for rHuEPO beta to induce ECG changes

A total of 24 adverse events were reported during the study; 14 events after rHuEPO reference compared to 10 events after rHuEPO biosimilar. However, the clinical relevance of this difference was considered negligible. Of the events with a suspected relationship headache, tiredness and dizziness were reported most frequently. The skin tolerability of both preparations was very good; the overall incidence of local reactions (bruising or inflammation) was $2.85 \%$.

During the 24 weeks of this trial anti-erythropoeitin antibodies were detected in two patients of the reference $\mathrm{rHuEPO}$ and none in the group of biosimilar rHuEPO.

\section{Discussion}

Renal anemia can be effectively managed by the administration of rHuEPO, which is able to increase hemoglobin levels and has been associated with significant improvements in the cardiovascular status of patients with CKD [15]. The administration of $\mathrm{rHuEPO}$ maintains adequate hemoglobin levels and avoids transfusions dependency in CKD patients.

Nowadays, there are three erythropoiesis-stimulating agents available on the Mexican market for the treatment of renal anemia; erythropoietin alpha, erythropoietin beta and darbepoetin alpha. Erythropoietin alpha and beta are both synthesized in Chinese hamster ovary cells and share the same amino acid sequence as endogenous EPO. On the contrary, darbepoetin alpha is biochemically different from endogenous EPO [16].

Erythropoiesis stimulating agents are effective in the management of anemia, but they substantially add to the overall treatment costs. Economizing treatment is, therefore, of significant importance for both payers and clinicians.

The introduction of biosimilars to the market is a controversial matter in nowadays. The two recombinant rHuEPO (reference and biosimilar) could be similar from the physicochemically point of view, but it is possible found some differences in the strains or manufacturing process that could have clinical significances in terms of safety and efficacy. The process - product dogma has been broken when the derived products mostly when they are highly purified and characterized. Actually there are several examples in the market in which similar recombinant proteins have shown safety and efficacy as interferons, insulins, somatropins $[17,18,19]$.

This was the first randomized clinical trial designed to compare the efficacy of rHuEPO beta manufactured by Roche with that of a biosimilar formulation. The results from this open study show that subcutaneously administered biosimilar
rHuEPO, was effective for the control of anemia in this group of CKD patients (hemodialysis) over 24 weeks. During the period of assessment hemoglobin levels were similar and maintained within the target range of $10-12$ grs as was reached with reference $\mathrm{rHuEPO}$ and also, in line with international recommendations [20]. The target hemoglobin range was defined according to recommendations in the current international literature. Over $90 \%$ of hemoglobin and $90 \%$ of hematocrit measurements were above the predefined lower limits of $10 \mathrm{~g} / \mathrm{dL}$ and $30 \%$, respectively in both groups. The increase of the hemoglobin concentrations after repeated doses of reference $\mathrm{rHuEPO}$ was $3.27 \mathrm{~g} / \mathrm{dL}$ and $3.55 \mathrm{~g} / \mathrm{dL}$ with biosimiliar rHuEPO, demonstrates that the effect on the hematopoiesis was comparable in both groups (21). Our findings are similar to those reported elsewhere in the literature [22,23]. As in the norm for biological variables, hemoglobin ranges exhibit a degree of variation with repeated measurement. In patients with CKD, this variability is even greater due to combination of factors, which include patient characteristics, intercurrent events and clinical practice and care patterns [24,25] With the recommended rHuEPO dose and optimal iron substitution, the increase of the hematocrit was $8.89 \%$ for reference $\mathrm{rHuEPO}$ and $8.07 \%$ for biosimilar rHuEPO. Differences between formulations were not statistically significant for any of the calculated parameters. In the present study, the rHuEPO beta dose was readjusted once monthly, in compliance with the regulations of ISSSTE, which established that hemodyalisis patients should undergo monthly hematocrit and hemoglobin measurements to be consistent with current medical practice. Variability in hemoglobin levels and in the number of patients outside recommended hemoglobin ranges might decrease if doses are adjusted more often [26].

The biosimilar rHuEPO and reference $\mathrm{rHuEPO}$ were well tolerated for the duration of the study. The frequency of signs and symptoms reported by patients during treatment and considered to be adverse effects was similar between the two groups. No thrombotic events (associated with vascular access) occurred during the course of the study. The most frequent events were headache, and hypertension as the same frequency and intensity reported by other authors $[27,28]$. Since the immune response point of view only two patients developed anti-erythropoietin antibodies with the reference $\mathrm{rHuEPO}$, without clinical significance.

From the clinician's point of view, studies in Mexican population are necessary for demonstrated the clinical safety and efficacy of rHuEPO biosimilar. This study indicates clearly a biosimilarity between both treatments and its subsequent cost minimizes treatment, potentially reflecting significant differences between health care system and centers. As the doses required to maintain stable hemoglobin are similar in both groups, the savings can be converted into cost reductions is an important reason for a clinician to switch to a biosimilar.

The data demonstrate that biosimilar rHuEPO is an acceptable alternative to reference rHuEPO. It would appear that patients can be transitioned from reference HuEPO to 
biosimilar rHuEPO at the same dose with no loss of control of anemia in base of the results shown in this study.

Our study has limitations, as was not blinded, the duration study and the number of patients; however the results are associated with relatively high hemoglobin levels without marked changes in dosing regimen

Our conclusion is the biosimilar product $\beta \mathrm{rHuEPO}$ is safe in clinical practice and is effective and stable in the tree times a week as well in frequency of application. Biosimilars offer a welcome opportunity to reduce treatment costs of renal anemia.

\section{Acknowledgements}

The study was funded by Laboratorios PISA S.A. de C.V. The analysis an interpretation of the data was performed by Investigación, Ciencia y Tecnología. The sponsor review the manuscript, but the final decision on content was with the main investigators.

\section{References}

[1] Brockmöller J, Köchling J, Weber W, et al. The pharmacokinetics and pharmacodynamics of recombinant human erythropoietin in haemodialysis patients. Br J Clin Pharmac 1992; 34: 499-508.

[2] Hsu CY, McCulloch CE, Curhan GC. Epidemiology of anemia associated with chronic renal insufficiency among adults in the United States: results from the Third National Health and Nutrition Examination Survey. J Am Soc Nephrol 2002; 13: 504-510.

[3] McClellan W, Aronoff SL, Bolton WK, et al. The prevalence of anemia in patients with chronic kidney disease. Curr Med Res Opin 2004; 20: 1501-1510.

[4] Besarab A, Bolton WK, Browne JK, et al. The effects of normal as compared with low hematocrit values in patients with cardiac disease who are receiving hemodialysis and epoetin. N Engl J Med 1998; 339: 584-590.

[5] Revicki Da, Brown RE, Feeney DH, et al. Health-related quality of life associated with recombinant human erythropoietin therapy for predialysis chronic renal disease patients. Am J Kidney Dis 1995; 25: 548-554.

[6] Collins AJ, Brenner RM, Ofman JJ; et al. Epoetin alfa use in patients with ESRD : an analysis of recent US prescribing patterns and hemoglobin outcomes. Am J Kidney Dis 2005; 46: 481-488.

[7] Mayer G, Thum J, Cada EM, et al. Working capacity is increased following recombinant human erythropoietin treatment. Kidney Int 1988; 34: 525-528.

[8] Macdougall IC, Lewis NP, Saunders MJ, et al. Long-term cardiorespiratory effects of amelioration of renal anemia by erythropoietin. Lancet 1990: 335: 489-493.

[9] Delano BG. Improvements in quality of life following treatment with $\mathrm{r}-\mathrm{HuEPO}$ in anemia hemodialysis patients. Am J Kidney Dis 1989; 14: 14-18.

[10] Evans RW, Rader B, Mannien DL, Cooperative Multicenter
EPO Clinical Trial Group. The quality of life of hemodialysis recipients treated with recombinant human erythropoietin. JAMA 1990; 263: 825-830.

[11] Ifudu O. Patient characteristics determining rHuEPO dose requirements. Nephrol Dial Transplant 2002; 17(5): 38-41.

[12] Stenvinkel P, Barany P. Anemia, rHuEPO resistance, and cardiovascular disease in end-stage renal failure: links to inflammation and oxidative stress. Nephrol Dial Transplant 2002; 17(5): 32-37.

[13] Zeid RJ. Regulatory and development issues in the demonstration of therapeutic equivalence for multisource biotechderivated pharmaceuticals. Drug Information Journal 2000; 34: 919-959.

[14] Schellekens H, Ryff JC. Biogenerics: the off-patient biotech products. Trends Pharmacol Sci 2002; 23: 119-121.

[15] Loughnan A, Ali GR, Abeygunasekara SC. Comparison of the therapeutic efficacy of epoetin beta and epoetin alfa in maintenance phase hemodialysis patients. REn Fail 2011: 33(3): 373-375.

[16] Storring PL, Tiplady RJ, Gaintes Das RE, et la. Epoetin alfa ans beta differ in their erythropoietin isoform compositions and biological properties. Br J Haematol 1998; 100: 79-89.

[17] Chamberlain P. Biogenerics: Europe takes another step forward while the FDA dives for cover. Drug Discov Today 2004; 9: 817-820.

[18] Hernandez-Bastida A, Meixueiro-Montes de Oca R. Equivalence on efficay and safety of two formulations of insulin glargine (biosimilar and reference) in the treatment of patients with type 2 diabetes. Clinical Medicine Research 2014; 3(2): 50-55.

[19] Palma-Aguirre J, Alvarez-Etchegaray S, Meixueiro-Montes de Oca R. Pharmacokinetic and pharmacodynamic biocomparability of two r-hGH (innovator and biosimilar) formulations after subcutaneous administration in healthy volunteers. Int J Pharm Pract \& Drug Res 2014; 4(1): 62-66.

[20] Locatelli F, Aljama P, Barany P, et al. Revised European best practice guidelines for the management of anemia in patients with chronic renal failure. Nephrol Dial Transplant 2004; 19(Suppl 2): 1-47.

[21] Furuland H, Linde T, Ahlmen $J$, et al. A randomised controlled trial of haemoglobin normalisation with epoetin alfa in pre-dyalisis and dialysis patients. Nephrol Dial Transplant 2003; 18(2): 353-361.

[22] Ebben JP, Gilbertson DT, Foley RN, Collins AJ. Hemoglobin level variability: associations with comorbidity, intercurrent events and hospitalizations. Clin J Am Soc Nephrol 2006; 1(6): 1205-1210.

[23] Berns JS, Elzein H, Lynn RI, et al. Hemoglobin variability in epoetin-treated hemodialysis patients. Kidney Int 2003; 64(4) 1514-1521.

[24] Breiterman-White R. Hemoglobin variability: management inevitable changes in clinical status. Nephrol Nurs J 2005; 32: 549-552.

[25] Fishbane S, Berns JS. Hemoglobin cycling in hemodialysis patients treated with recombinant human erythropoietinKidney Int 2005; 68(3): 1337-1343. 
[26] Fishbane S, Berns JS,. Evidence and implications of hemoglobin cycling in anemia management. Nephrol Dial Transplant 2007; 22(8): 2129-2132.

[27] Jacquot C, Ferragu-Haguet M, Lefebvre A, et al. Recombinant erythropoietin and blood pressure. Lancet 1987; 2: 1083-1085.
[28] Klinkmann H, Wieczorek L, Scigalla P. Adverse events of subcutaneous recombinant human erythropoietin therapy: results of a controlled multicenter European study. Artif Organs 1993; 17: 219-225. 